\title{
INITIAL RESULTS OF AORTIC VALVE RECONSTRUCTION USING AUTOLOGOUS PERICARDIUM (OZAKI'S PROCEDURE)
}

\author{
Ngo Thanh Hung ${ }^{1+*}$, Nguyen Cong Huu ${ }^{1+}$, Tran Thuy Nguyen ${ }^{1+}$, Nguyen Hoang Nam ${ }^{1}$, \\ Nguyen Ngoc Anh ${ }^{1}$, Luong Hoang Long ${ }^{2}$, Do Duc Thinh ${ }^{1}$, Le Ngoc Thanh ${ }^{1}$, Doan Quoc Hung ${ }^{3}$
}

\section{ABSTRACT}

Introduction: This study report the clinical characteristics, surgical indications, surgical technique and initial outcomes of autologous pericardial aortic valve reconstruction using Ozaki's procedure.

Methods: The study included consecutive patients with isolated aortic valve disease who underwent Ozaki's procedure between June 2017 and December 2019. Aortic valve cups were reconstructed by autologous pericardium using Ozaki's procedure.

Results: Seventy-two patients were enrolled (mean age $52.9 \pm 13$ years; 53 males) and consisted of 30 aortic stenosis cases, 20 aortic regurgitation cases, and 22 patients with a combination of both 72 patients, a bicuspid aortic valve was present in 20, and 7 patients had infective endocarditis. Surgery was performed via a full or partial sternotomy. The procedure was successful in 70 case, and two patients were converted to prosthetic valve replacement. The aortic cross-clamp time was $106.3 \pm 13.8$ minutes, cardiopulmonary bypass time was $136.7 \pm 18.5$ minutes. One patient died of cardiac tamponade in hospital, and two patients underwent reoperation due to bleeding and sternal infection, respectively, were observed during the follow-up period of 30 days. 1-month postoperative echocardiography revealed that one patient had moderate aortic valve regurgitation, max transvalvular pressure gradient was $16.1 \pm 2.3 \mathrm{mmHg}$, and aortic valve area was $2.5 \pm 0.2 \mathrm{~cm}^{2}$.

Conclusions: Aortic valve reconstruction using autologous pericardium by Ozaki's procedure was feasible, good hemodynamics, and can be applied to all lesions of the aortic valve.

Keywords: Aortic valve insufficiency, aortic valve stenosis, cardiac surgical procedures, pericardium, reconstructive surgical procedures.

\section{INTRODUCTION}

Aortic valve disease (AVD) is a common valvular disorder with a prevalence of more than $2 \%$ in elderly patients [1][2]. The most common causes of AVD are degeneration, calcification, congenital defects, post-rheumatic fever and infection [3][4][5]. Treatments for severe AVD include aortic valve replacement surgery (AVRS), aortic valve repair and transcatheter aortic valve implantation (TAVI) [6], in which AVRS with one prosthetic valve used widely in cardiovascular surgery centers today has been still the gold standard [7]. However, AVRS has several limitations such as: mechanical prosthetic valves required life-long anticoagulant treatment which is associated with the risk of major bleeding or prosthetic valve thrombosis [8][9]. Bioprosthetic valves are also

\footnotetext{
1 Departement of Cardiothoracic Surgery- Cardiovascular Center- E Hospital

2 Department of Allergy-Immunology and Dermatology, E hospital

3 Cardiovascular and Thoracic Center- Viet Duc Hospital

+These authors contributed equally to this work

*Corresponding authors: Doan Quoc Hung \& Ngo Thanh Hung

Email: hung.doanquoc@gmail.com \& ngohunghmu@gmail.com

Address: E Hospital, 87-89 Tran Cung, Hanoi, Vietnam, 10000

Vietnam National University, 114 Xuan Thuy, Hanoi,

Vietnam, 10000
} 
associated with valve degeneration, calcification, or immune reactions [10][11]. Furthermore, the hemodynamics of prosthetic valves are inferior to those of the native valves [12]. Therefore, the number of aortic valve repair/reconstruction surgery has been increased remarkably. The surgical technique includes annular plication, patch valve perforation, and cusp expansion [13].

The first Ozaki surgery was performed in Toho Hospital, in Tokyo, Japan in 2007 [14]. Today, this technique, which has been used in many countries with more than 4000 patients undergoing surgery successfully [15], has many reported advantages including no need anticoagulant, a low pressure gradient, a high effective valvular orifice area, and preservation of aortic root physiology. In Vietnam, prosthetic aortic valve replacement has been continued to use as standard procedure, whereas Ozaki's procedure is still considered as a new technique [16]. In addition, there have not been many reports on this technique, thence we conducted this study for two objectives (a) to describe the clinical and echocardiographic changing features before and after surgery, indications and technical procedures of the Ozaki's procedure, (b) to report early results of aortic valve reconstruction using Ozaki's procedure.

\section{METHODS}

\section{Patients}

All patients had diagnosis of isolated AVD underwent aortic valve reconstruction with autologous pericardium using Ozaki's procedure from June 2017 to December 2019 at Cardiovascular Center, E Hospital. The indications for aortic valve reconstruction by Ozaki's procedure consisted of (1) isolated AVD with aortic surgical indications and (2) the patient consented to be performed by operation using Ozaki method. We excluded all of patients who had history of heart surgery, emergency surgery, and other valvular disease [17].

\section{Operation technique}

All patients underwent surgery under general anesthesia. A central venous line was established, and invasive blood pressure monitoring was performed. Other preoperative procedures were similar to those used in open heart surgery. All patients underwent transesophageal echocardiography. The aortic valve was reconstructed according to the techniques described by Ozaki and colleagues [12]. Sternotomy or a minimally invasive J- or Lshape sternotomy (upper half) was performed. The pericardium was dissected and harvested using a Harmonic knife $(8 \quad x \quad 8 \quad c m)$. The diaphragmatic face was preferred as it is thicker than other parts of the pericardium. The harvested pericardium was treated with $0.6 \%$ glutaraldehyde for $10 \mathrm{~min}$ and washed with normal saline for $6 \mathrm{~min}$. This step was repeated three times (18 $\mathrm{min}$ in total). After processing the pericardium, cardiopulmonary bypass was instituted with one arterial and one venous cannula. The left heart suction line ran from the right upper pulmonary vein through the mitral valve to the left ventricle. Cardioplegia was delivered through the aortic root or directly through the coronary orifice, and body 
temperature was reduced to $32^{\circ} \mathrm{C}$. The aorta was opened along the circumference $1.5 \mathrm{~cm}$ above the right coronary root. All leaflets of the aortic valve were removed. The size of the aortic valve annulus was measured using a prosthetic valve measuring tool, and the distance between the commissures were measured using an AVNeo kit [15]. The pericardium was cut to form three leaflets using Ozaki's block based on the measured size, prioritizing the largest leaflet at the diaphragm side. The leaflets were sutured to the annulus using 4/ 0 suture. The 3 respective commissures were reconstructed. We reconstructed bi-leaflets with bicuspid type 0 (Sievers) [18] and reconstructed tri-leaflets with bicuspid type I.

\section{Statistic}

Preoperative demographic, clinical, laboratory, echocardiographic, and electrographic data were collected. Operative parameters, including operative time, extracorporeal circulation time, aortic cross-clamp time, size of the aortic valve annulus, cups's size, and number of reconstructed leaflets, were also collected. After the surgery, the time of ventilation, length of stay in the intensive care unit, and surgical complications such as bleeding, reoperation, renal failure, and pneumonia were recorded. Transthoracic echocardiography was performed on 1 week and in 1 month after surgery, and all echocardiographic parameters were recorded. Categorical data are presented as frequencies and percentages while continuous variables are presented as mean standard deviation if normally distributed, or median and interquartile range otherwise. All analyses were performed using software, and a two-tailed $\mathrm{p}$ value $<0.05$ was considered statistically significant.

\section{Ethics committee}

The study was approved by the research committee of E Hospital and Hanoi Medical University (number NCS 06/HMUIRB).

\section{RESULTS}

Clinical and paraclinical preoperative characteristics were shown in Table 1

Intraoperative characteristics were shown in Table 2

Complications were shown in Table 3

1-month postoperative echocardiographic characteristics were shown Table 4

\section{DISCUSSION}

The mean age in our study was 53 years old (table 1), which is similar to the study of Krane et al 17 (54 years old), but lower than in the study of Ozaki et al 18 (71 years old). This can be explained by the causes of the AVD in the Ozaki's study were degeneration and calcification in the elderly. By contrast, the common causes in our study consisted of calcification, postrheumatic fever, bicuspid valve and endocarditis, which results in mean age in our study was lower than in others..

In this study, the cardiac risk factors included hypertension (47.2\%), diabetes (2.8\%), dyslipidemia (5.6\%), smoking(51.4\%) (table 1). The ratio of cardiac risk factors was lower in the study of Ozaki et al [18], Oliver et al [19]. According to Stewart et al [20], the mechanism of aortic valve disease caused by factors such as hypertension, diabetes, and dyslipidemia is the same as that of atherosclerosis. 
The common clinical symptoms were shortness of breath (NYHA II-III), angina (CCS II) was found in $47.2 \%$ of cases (table 1). Luca et al [21] showed NYHA II-III was the most common (77\% of cases), angina CCS I-II was found in $94 \%$ of cases. Angina in AVD was due to increased myocardial oxygen demand and decreased coronary flow. According to Carabello et al [22], the mortality rate increases by $25 \%$ per year when angina was presented.

Ozaki et all [23] indicated aortic valve reconstruction surgery using autologous pericardium for all aortic valve lesions: stenosis, regurgitation, and combination of both. The procedure can be performed to all patients with different ages and valve morphology [23],[24]. In our study, autologous pericardial aortic valve reconstruction using Ozaki's procedure was indicated for patients had isolated AVD and age from 19 to 79 years olds. During emergency condition, we chose aortic prosthetic valve replacement in order to reduce cardiopulmonary bypass time and aortic cross-clamp time.

In this study, 7 cases were performed by minimally invasive surgery with upper ministernotomy revealed postoperative results found a no difference from those with full sternotomy (table 2). Nguyen Hoang Dinh et al reported successful outcomes of minimally invasive aortic reconstruction using Ozaki's procedure [25]. When comparing 30 patients with minimally invasive surgery among all 140 patients who underwent Ozaki surgery, Rosseikin et al showed the amount of bleeding in minimally invasive surgery was inferior to open surgery [26]. In our view, minimally invasive operation, however, should be performed by surgeons experienced in dissecting and treating pericardium, which ensures pericardium wasn't damaged.

We had 2 patients after autologous pericardial aortic reconstruction had to be converted to prosthetic valve replacement (table 2). Ozaki et al revealed that no conversion to valve replacement in all 850 aortic reconstructed patients [23]. Of 103 patients had Ozaki's technique, there were 2 patients required to convert into replacing prosthetic valve due to occlusion of left coronary artery caused by left cusp according to Krane et al [27]. Therefore, the measurement of distance among 2 commissures should be ensured to be stable and tight, which prevents cusps s from being oversized.

There was a 1-day postoperative death caused by acute cardiac tamponade accounted for $1.4 \%$ of all cases (table 1 ). In comparison, 30-day mortality rate reported by Ozaki and Oliver was $1.8 \%$ and $3.3 \%$, respectively [19][23]. Besides, the early mortality rate observed in aortic reconstruction operation using Ozaki procedure was similar to aortic valve replacement in a range of 1 to 3 percent.

Follow-up 1 month after surgery, only one patient had a moderate aortic valve regurgitation which has been conserved to management (table 4). Hemodynamic aortic valve characteristics (max trans-valvular pressure gradient was $16.1 \mathrm{mmHg}$, AVA was $2.5 \mathrm{mmHg}$ ) found similar results to other studies, in which maximum pressure gradient according to Luca's study and Ozaki's study was $20 \mathrm{mmHg}$ and $19,5 \mathrm{mmHg}$, respectively [21]'[23]. Hemodynamics of aortic valve after reconstruction using Ozaki's procedure was favorable because cusps was stitched directly natural annulus without effects 
on it, thus it did not reduce effective area of valve. This technique preserved the compliance of the annulus and aortic root during systole, which lead to increase the valve area more.

\section{CONCLUSION}

Aortic valve reconstruction using Ozaki's procedure could be implemented for all of aortic valve lesions in a wide range of age. The operation can be performed by minimally invasive incision resulting in satisfactory initial outcomes. Mortality rate and complications were similar to aortic valve replacement, and hemodynamic parameters of the valves were favorable.

\section{Limitations}

This single-center study was conducted in a small quantity of patient for relatively short term. Therefore, the study on a larger number of patients should be undertaken in a combination of multiple centers.

\section{REFERENCES}

1. Manning WJ. Asymptomatic aortic stenosis in the elderly: A clinical review. JAMA - Journal of the American Medical Association. 2013;310(14):1490-7.

2. Czarny MJ, Resar JR. Diagnosis and management of valvular aortic stenosis. Clinical Medicine Insights: Cardiology. 2014;8:15-24.

3. Iung B, Baron G, Butchart EG, Delahaye F, Gohlke-Bärwolf C, Levang OW, et al. A prospective survey of patients with valvular heart disease in Europe: The Euro Heart Survey on valvular heart disease. European Heart Journal. 2003;24(13):1231-43.

4. Roberts WC, Ko JM. Frequency by decades of unicuspid, bicuspid, and tricuspid aortic valves in adults having isolated aortic valve replacement for aortic stenosis, with or without associated aortic regurgitation. Circulation. 2005;111(7):920-5.

5. Otto CM, Prendergast B. Aortic-valve stenosis From patients at risk to severe valve obstruction. New England Journal of Medicine. 2014;371(8):744-56.

6. Nishimura RA, Otto CM, Bonow RO, Iii JPE, Fleisher LA, Jneid H, et al. 2017 AHA / ACC Focused Update of the 2014 AHA / ACC Guideline for the Management of Patients With Valvular Heart Disease A Report of the American College of Cardiology / American Heart Association Task Force on Clinical Practice Guidelines. 2017. 1159-1195 p.

7. Vahanian A, Alfieri $\mathrm{O}$, Andreotti $\mathrm{F}$, Antunes MJ, Barón-Esquivias $\mathrm{G}$, Baumgartner $\mathrm{H}$, et al. Guidelines on the management of valvular heart disease (version 2012). European Heart Journal. 2012;33(19):2451-96.

8. North RA, Sadler L, Stewart AW, McCowan LME, Kerr AR, White HD. Long-term survival and valve-related complications in young women with cardiac valve replacements. Circulation. 1999;99(20):2669-76.

9. Cannegieter SC, Rosendaal FR, Briët E. Thromboembolic and bleeding complications in patients with mechanical heart valve prostheses. Circulation. 1994;89(2):635-41.

10. Khan SS, Trento A, DeRobertis M, Kass RM, Sandhu M, Czer LSC, et al. Twenty-year comparison of tissue and mechanical valve replacement. Journal of Thoracic and Cardiovascular Surgery. 2001;122(2):257-69.

11. Human P, Zilla P. Characterization of the immune response to valve bioprostheses and its role in primary tissue failure. Annals of Thoracic Surgery. 2001;71(5 SUPPL.):20-3.

12. Ozaki S, Kawase I, Yamashita H, Uchida S, Nozawa Y, Takatoh M, et al. A total of 404 cases of aortic valve reconstruction with glutaraldehyde- 
treated autologous pericardium. Journal of Thoracic and Cardiovascular Surgery. 2014;147(1):301-6.

13. Halees $\mathrm{Z}$ Al, Shahid $M$ Al, Sanei A Al, Sallehuddin A, Duran C. Up to 16 years follow-up of aortic valve reconstruction with pericardium: A stentless readily available cheap valve? European Journal of Cardio-thoracic Surgery. 2005;28(2):200-5.

14. Ozaki S, Kawase I, Yamashita H, Uchida S, Nozawa $\mathrm{Y}$, Matsuyama $\mathrm{T}$, et al. Aortic valve reconstruction using self-developed aortic valve plasty system in aortic valve disease. Interactive Cardiovascular and Thoracic Surgery. 2011;12(4):550-3.

15. https://avneo.net.

16. Ngo HT, Nguyen HC, Nguyen TT, Le TN, Camilleri L, Doan HQ. Reconstruction of aortic valve by autologous pericardium (Ozaki's procedure): Single center experience in Vietnam. Asian Cardiovascular and Thoracic Annals. 2020;(1).

17. Nishimura RA, Otto CM, Bonow RO, Iii JPE, Fleisher LA, Jneid H, et al. 2017 AHA / ACC Focused Update of the 2014 AHA / ACC Guideline for the Management of Patients With Valvular Heart Disease A Report of the American College of Cardiology / American Heart Association Task Force on Clinical Practice Guidelines. 2017. 1159-1195 p.

18. Sievers HH, Schmidtke C. A classification system for the bicuspid aortic valve from 304 surgical specimens. Journal of Thoracic and Cardiovascular Surgery. 2007;133(5):1226-33.

19. Reuthebuch O, Koechlin L, Schurr U, Grapow M, Fassl J, Eckstein FS. Aortic valve replacement using autologous pericardium: single centre experience with the Ozaki technique. Swiss medical weekly. 2018;148(February):w14591.

20. Stewart BF, Siscovick D, Lind BK, Gardin JM, Gottdiener JS, Smith VE, et al. Clinical factors associated with calcific aortic valve disease. Journal of the American College of Cardiology. 1997;29(3):630-4.

21. Koechlin L, Schurr U, Miazza J, Imhof S, Maurer $\mathrm{M}$, Erb J, et al. Echocardiographic and Clinical Follow-up After Aortic Valve Neocuspidization Using Autologous Pericardium. World Journal of Surgery. 2020;44(9):3175-81.

22. Carabello BA, Paulus WJ. Aortic stenosis. The Lancet. 2009;373(9667):956-66.

23. Ozaki S, Kawase I, Yamashita H, Uchida S, Takatoh M, Kiyohara N. Midterm outcomes after aortic valve neocuspidization with glutaraldehydetreated autologous pericardium. Journal of Thoracic and Cardiovascular Surgery. 2018;155(6):2379-87.

24. Ozaki S, Kawase I, Yamashita H, Uchida S, Nozawa Y, Takatoh M, et al. Reconstruction of bicuspid aortic valve with autologous pericardium - Usefulness of tricuspidization. Circulation Journal. 2014;78(5):1144-51.

25. Nguyen DH, Vo AT, Le KM, Vu TT, Nguyen TT, $\mathrm{Vu} \mathrm{TT}$, et al. Minimally Invasive Ozaki Procedure in Aortic Valve Disease: The Preliminary Results. Innovations: Technology and Techniques in Cardiothoracic and Vascular Surgery. 2018;13(5):332-7.

26. Rossě̌kin E V., Kobzev EE, Bazylev V V. Minimally invasive Ozaki technique. Angiology and vascular surgery. 2019;25(3):142.

27. Krane M, Boehm J, Prinzing A, Ziegelmueller J, Holfeld J, Lange R. Excellent Hemodynamic Performance After Aortic Valve Neocuspidization Using Autologous Pericardium. Annals of Thoracic Surgery. 2021;111(1):126-33. 
Table 1. Preoperative baseline characteristics

\section{Characteristics}

Value

Age, mean $\pm \mathrm{SD}$, years

$52.9 \pm 13.8$

Male, $\mathrm{n}(\%)$

Body mass index, mean \pm SD

$22.1 \pm 2.9$

Cardiac risk factors

Hypertension, $\mathrm{n}(\%)$

Diabetes

Hyperlipidemia

Smoking

Atrial fibrillation

Dyspnea (NYHA)

$\mathrm{I}, \mathrm{n}(\%)$

II, $\mathrm{n}(\%)$

26(36.1)

III, $\mathrm{n}(\%)$

$37(51.4)$

IV, $\mathrm{n}(\%)$

$6(8.3)$

Angia (CCS)

I, $n(\%)$

27(37.5)

II, $\mathrm{n}(\%)$

$30(41.7)$

III, $\mathrm{n}(\%)$

13(18.1)

IV, $\mathrm{n}(\%)$

Syncope, $\mathrm{n}(\%)$

Fever, n(\%)

Valve morphology

Tricuspid, n(\%)

Bicuspid, n(\%)

Aortic valve disease

Isolated aortic stenosis, $\mathrm{n}(\%)$

Aortic regurgitation, $\mathrm{n}(\%)$

Combined, $\mathrm{n}(\%)$

$22(30.5)$

$\mathrm{LVEF}$, mean $\pm \mathrm{SD}, \%$

$58.2 \pm 7.1$ 
Table 2. Intraoperative parameters

Characteristics

Value

No. of reconstructed leaflets

$$
\begin{aligned}
& 1, \mathrm{n}(\%) \\
& 2, \mathrm{n}(\%) \\
& 3, \mathrm{n}(\%)
\end{aligned}
$$

Aortic valve annulas size, mean $\pm \mathrm{SD}, \mathrm{mm}$

$<21 \mathrm{~mm}, \mathrm{n}(\%)$

Aortic cross-clamp time, mean $\pm \mathrm{SD}$, minutes

$106.3 \pm 13.8$

Cardiopulmonary bypass time, mean $\pm \mathrm{SD}$, minutes

$136.7 \pm 18.5$

\section{Table 3. Postoperative characteristics}

\section{Characteristics}

Value

Mechanical ventilation time, mean $\pm \mathrm{SD}$, minutes

$11.3 \pm 7.1$

$>24$ hours, $n(\%)$

Death, $\mathrm{n}(\%)$

Reoperation

Bleeding, $\mathrm{n}(\%)$

Sternal infection, $\mathrm{n}(\%)$

Pneumonia, $\mathrm{n}(\%)$

New atrial fibrilation, $\mathrm{n}(\%)$

Acute kidney injury, $\mathrm{n}(\%)$

$4.1 \pm 1.9$

Table 4. 1-month postoperative echocardiography characteristics.

Characteristics

Value

Aortic valve regurgitation

None, $\mathrm{n}(\%)$

Mild, $\mathrm{n}(\%)$

Moderate, $\mathrm{n}(\%)$

Severe, $\mathrm{n}(\%)$

Aortic valve stenosis, $\mathrm{n}(\%)$

Maximum pressure gradient, mean $\pm \mathrm{SD}, \mathrm{mmHg}$

$16.1 \pm 2.3$

Mean pressure gradient, mean $\pm \mathrm{SD}, \mathrm{mmHg}$

$7.4 \pm 1.5$

Aortic valve Area, mean $\pm \mathrm{SD}, \mathrm{cm}^{2}$

$2.5 \pm 0.2$

LVEF, mean $\pm \mathrm{SD}, \%$

$59.2 \pm 3.5$ 